\title{
Distribuição probabilística do fator de escala de dois solos do Estado da Paraíba ${ }^{1}$
}

Antonio C. D. Antonino', Carlos F. Ruiz², Eduardo S. de Souza ${ }^{3}$, André M. Netto ${ }^{1} \&$ Rafael Angulo-Jaramillo ${ }^{4}$

\author{
1 Depto. de Energia Nuclear, UfPE, Av. Prof. Luiz Freire 1000. CEP 50740-540, Recife, PE.. Fone (81) $2126-8252$. \\ Fax: (81) 21268250. E-mail: acda@ufpe.br. (Foto) \\ 2 IMTA, Paseo Cuauhnáhuac, 85326, 2550. Progreso, Morelos, México. E-mail: cfuentes@tlaloc.imta.mx. \\ 3 PROTEN, Depto. de Energia Nuclear, UFPE. Fax: (81) 3271-8250. \\ ${ }^{4}$ CNRS, LTHE (UMR 5564 CNRS, INPG, IRD, UJF), BP 53, 38041 Grenoble cedex 9, França. E-mail: angulo@hmg.inpg.fr.
}

Protocolo 95 - 9/7/2002 - Aprovado em 18/2/2004

\begin{abstract}
Resumo: A distribuição probabilística do fator de escala da camada superficial, considerando-se a teoria dos meios fractais auto-similares, foi determinada para dois solos do Estado da Paraíba. 0 estudo foi realizado em duas áreas experimentais, com distintas condições de clima e solo, de aproximadamente 4 ha. A primeira na Fazenda Várzea Grande, no município de São João do Cariri, PB $\left(7^{\circ} 23^{\prime} \mathrm{S}\right.$ e $\left.36^{\circ} 31^{\prime} \mathrm{O}\right) \mathrm{com}$ o solo classificado como Neossolo Flúvico enquanto a segunda, se localiza no Centro de Ciências Agrárias, da UFPB, no município de Areia, PB (65ㅇ's, $35^{\circ} 41^{\prime}$ ) $)$, com o solo classificado como Latossolo Amarelo. Para estimar a condutividade hidráulica à saturação do solo, realizaram-se ensaios de infiltração com infiltrômetro de anel (15 cm de diâmetro) na superfície do solo, em 80 pontos distribuídos regularmente em uma malha de $25 \times 25 \mathrm{~m}$. Para ambos os solos, o logaritmo dos fatores de escala que intervém nos fractais auto-similares segue uma distribuição de Gauss ou distribuição normal. A função de distribuição probabilística permitiu estimar a dimensão fractal do meio poroso, que representa os solos estudados.
\end{abstract}

Palavras-chave: fator de escala, condutividade hidráulica, dimensão fractal

\section{Probabilistic distribution of the scale factor of two soils in the State of Paraíba - Brazil}

\begin{abstract}
The probabilistic distribution of the scale factor of superficial layer was determined, considering the similar fractal theory for two soils in the Paraíba State. The study was performed at two experimental sites, with different climate and soil conditions of approximately 4 ha. The first was located in the Várzea Grande farm, in the municipality of São João do Cariri, PB $\left(7^{\circ} 23^{\prime} \mathrm{S}\right.$ e $\left.36^{\circ} 31^{\prime} \mathrm{O}\right)$. The soil was classified as Fluvent. The second was located in the Centro de Ciências Agrárias, of the UFPB, in the municipality of Areia, PB (6 ${ }^{\circ} 58^{\prime} \mathrm{S}, 35^{\circ} 41^{\prime} \mathrm{W}$ ). The soil was classified as Oxisol. To estimate the soil saturated hydraulic conductivity, infiltration experiments were performed using a ring infiltrometer $(15 \mathrm{~cm}$ diameter) on the soil surface, at 80 points on a regular mesh of $25 \times 25 \mathrm{~m}$. For both soils, the logarithms of the scale factors followed a Gaussian distribution or normal distribution. The probabilistic distribution function allowed the estimation of the fractal dimension of the porous media that represent the studied soils.
\end{abstract}

Key words: scale factor, hydraulic conductivity, fractal dimension

\section{INTRODUÇÃO}

A condutividade hidráulica da camada superficial do solo desempenha papel importante na partição da quantidade de água, seja de precipitação pluviométrica ou de irrigação, que atinge o solo em infiltração e/ou escoamento superficial.

Diferentes trabalhos reportados na literatura (Nielsen et al., 1973; Warrick \& Amoozegard-Fard, 1979; Vauclin, 1982; Hopmans,1987) mostram que a condutividade hidráulica, em especial seu valor - a saturação $\left(\mathrm{K}_{\mathrm{s}}\right)$, apresenta grande variabilidade no espaço devido à própria variabilidade espacial da geometria interna do meio poroso, o que tem conduzido a se estudar a condutividade hidráulica como uma variável estocástica, isto é, com as ferramentas da estatística matemática e particularmente da geoestatística. Em trabalhos recentes, têmse utilizado também os conceitos da geometria fractal para o estudo desta variabilidade espacial (Namuche et al., 1996). 
A teoria dos meios similares, proposta por Miller \& Miller (1956), simplifica consideravelmente o estudo da variabilidade espacial das características hidrodinâmicas do solo e foram eles os primeiros a observar a invariância de escala das equações do movimento da água em um meio poroso. Nesta teoria, considera-se que a geometria interna de um meio poroso qualquer em uma região, pode ser obtida a partir de um solo de referência por intermédio de um fator de similaridade ou fator de escala.

A utilização de modelos estocásticos da dinâmica da água no solo necessita do conhecimento, a priori, da lei de distribuição, caracterizando a variabilidade dos parâmetros aleatórios do modelo (Braud et al., 1995). O escalonamento é uma abordagem atrativa para expressar a variabilidade das propriedades hidráulicas do solo, cuja vantagem reside no fato de que ela possibilita a modelagem através de um único parâmetro de significação física, ou seja, o fator de escala.

O objetivo do presente trabalho é o estudo da distribuição probabilística do fator de escala da camada superficial, considerando a teoria dos meios fractais auto-similares, de dois solos do estado da Paraíba.

\section{TEORIA}

As características hidrodinâmicas, isto é, a curva de retenção da água no solo, $\theta(\psi)$, e a condutividade hidráulica em função da pressão da água do solo, $\mathrm{K}(\psi)$ são relacionadas com a geometria interna do solo por intermédio das leis de Laplace e de Poiseuille. A lei de Laplace que relaciona a pressão da água do solo, $\psi$, com um raio de poro, é dada por:

$$
\psi=-\frac{2 \sigma \cos \alpha}{\rho_{w} g R}
$$

sendo $\sigma$ a tensão na interface água-ar $\left[\mathrm{kg} \mathrm{s}^{-2}\right] ; \rho_{\mathrm{w}}$ a massa específica da água $\left[\mathrm{kg} \mathrm{m}^{-3}\right]$; g a aceleração da gravidade [m $\mathrm{s}^{-2}$; $\mathrm{R}$ o raio do capilar (poro) [m] e $\alpha$ o ângulo de contato formado entre o menisco de água e as partículas sólidas.

A lei de Poiseuille, que relaciona a velocidade média (v) num capilar cilíndrico de raio $\mathrm{R}$ e o gradiente hidráulico, é expressa por:

$$
v=-\frac{\rho_{w} g}{8 \mu_{c}} R^{2} \operatorname{grad}(H)
$$

em que $\mathrm{H}=\psi+\mathrm{z}$ é o potencial hidráulico [m] igual à soma do potencial de pressão da água no solo $(\psi)$ (negativo na zona não saturada e positivo na zona saturada, expresso como a altura equivalente de coluna de água) e do potencial gravitacional assimilado à coordenada espacial vertical $(\mathrm{z})$; $\mu_{\mathrm{c}}$ é o coeficiente de viscosidade dinâmica $\left[\mathrm{kg} \mathrm{m}^{2} \mathrm{~s}^{-1}\right]$. A partir dessas leis, pode-se estabelecer as relações entre a pressão da água, $\psi$, e a condutividade hidráulica, K, com um raio de poro característico:

$$
\psi=\frac{\mathrm{C}_{1}}{\mathrm{R}} \quad \mathrm{K}=\mathrm{C}_{2} \mathrm{R}^{2}
$$

sendo $\mathrm{C}_{1}$ e $\mathrm{C}_{2}$ coeficientes de proporcionalidade.

\section{Teoria dos meios similares}

A teoria dos meios similares (Miller \& Miller, 1956) considera que a geometria interna de um meio poroso qualquer em uma região pode ser obtida a partir de um solo de referência por intermédio de um fator de similaridade ou fator de escala, $r$ :

$$
r=\frac{R}{R_{*}}
$$

em que $\mathrm{R}$ é o raio de poro de um solo qualquer e $\mathrm{R}_{*}$ é o raio do poro correspondente, no solo de referência.

$\mathrm{Na}$ teoria dos meios similares assume-se implicitamente que a porosidade é a mesma em todos os meios. Esta conseqüência não é tão forte se a porosidade é construída de maneira adequada (Fuentes, 1992).

A partir das Eqs. (3) e (4) e se considerando constantes a tensão superficial, a viscosidade e a densidade da água, obter-se-ão as relações seguintes entre as características hidrodinâmicas de um solo dado e as do solo de referência (*):

$$
\psi=\frac{\psi^{*}}{\mathrm{r}} \quad \mathrm{K}=\mathrm{K}^{*} \mathrm{r}^{2}
$$

As relações anteriores se generalizam a outras propriedades hidrodinâmicas (W) pela expressão:

$$
\mathrm{W}=\mathrm{r}^{\mathrm{p}} \mathrm{W}^{*}
$$

$\mathrm{p}=-1$ para os potencias de pressão $(\psi)$ e gravitacional $(\mathrm{z}) \mathrm{e}$ para as coordenadas espaciais $(\mathrm{x}, \mathrm{y}, \mathrm{z}) ; \mathrm{p}=-3$ para o tempo; $\mathrm{p}=2$ para a condutividade e para o fluxo.

De posse de observações da condutividade saturada em $\mathrm{N}$ solos $(\mathrm{j}=1,2, \ldots, \mathrm{N})$, o solo de referência pode ser construído, de acordo com Fuentes (1992), da seguinte maneira: aplicando a Eq. 5 à condutividade saturada do solo j-ésimo, obtendo-se somatórios dos logaritmos e se assimilando a condutividade do solo de referência a média geométrica, obtem a expressão abaixo:

$$
\ln \left(\mathrm{K}_{\mathrm{s}}^{*}\right)=\frac{1}{\mathrm{~N}} \sum_{\mathrm{j}=1}^{\mathrm{N}} \ln \left(\mathrm{K}_{\mathrm{sj}}\right) ; \mu_{\tau}=\frac{1}{\mathrm{~N}} \sum_{\mathrm{j}=1}^{\mathrm{N}} \ln \left(\mathrm{r}_{\mathrm{j}}\right)=0
$$

sendo $\mu_{\tau}$ a média aritmética dos logaritmos dos fatores de escala: $\tau=\ln (\mathrm{r})$. Os fatores de escala se estimam através da Eq. 5 aplicada às condutividades saturadas:

$$
r_{j}=\sqrt{\frac{K_{s j}}{K_{s}^{*}}}
$$

\section{Distribuição probabilística dos fatores de escala}

$\mathrm{Na}$ literatura, tem-se que os fatores de escala seguem uma distribuição probabilística (e.g. Nielsen et al., 1973). Posto que $r \geq 0$, a função densidade deve ser zero para $r<0$. Se funções 
de densidades definidas sobre toda a reta real são utilizadas, é conveniente introduzir a transformação logarítmica dos fatores de escala, isto é: $-\infty<\tau=\ln (\mathrm{r})<\infty$. Se $\bar{\omega}(\mathrm{r})$ e $\mathrm{p}(\tau)$ representam, respectivamente, as funções de densidade dos fatores de escala e seus logaritmos, então :

$$
\mathrm{p}(\tau)=\mathrm{r} \varpi(\mathrm{r})
$$

A probabilidade acumulada é definida por :

$$
\mathrm{F}(\tau)=\int_{-\infty}^{\tau} \mathrm{p}(\bar{\tau}) \mathrm{d} \bar{\tau}=\int_{0}^{\mathrm{r}} \bar{\Phi}(\overline{\mathrm{r}}) \mathrm{d} \overline{\mathrm{r}}=\omega(\mathrm{r})
$$

O valor esperado de $\mathrm{f}(\mathrm{r})$ é definido por:

$$
E\{f(r)\}=\int_{0}^{\infty} f(r) \omega(r) d r=\int_{-\infty}^{\infty} f\left(e^{\tau}\right) p(\tau) d \tau=E\left\{f\left(e^{\tau}\right)\right\}
$$

A função densidade $\mathrm{p}(\tau)$ de Gauss (log-normal) descreve satisfatoriamente a densidade do logaritmo dos fatores de escala (Nielsen et al., 1973; Warrick \& Amoozegard-Fard, 1979; Vauclin, 1982; Fuentes, 1992). A função de distribuição gaussiana e a função de probabilidade, obtidas da Eq. 11 são, respectivamente:

$$
\begin{aligned}
& \mathrm{p}(\tau)=\frac{1}{\sigma_{\tau} \sqrt{2 \pi}} \exp \left[-\frac{\tau^{2}}{2 \sigma_{\tau}^{2}}\right] \\
& \mathrm{F}(\tau)=\frac{1}{2}\left[1+\operatorname{erf}\left(\frac{\tau}{\sigma_{\tau} \sqrt{2}}\right)\right]
\end{aligned}
$$

cuja média é considerada zero $\left(\mu_{\tau}=0\right)$ de acordo com a normalização dada pela Eq. 7; $\sigma_{\tau}$ é o desvio padrão do logaritmo dos fatores de escala e erf(x) denota a função erro.

Fazendo $\mathrm{f}(\mathrm{r})=\mathrm{r}^{\mathrm{n}}$ na Eq. 11 e considerando a Eq. 12, obter-se-ão os momentos de ordem $\mathrm{n}$ dos fatores de escala:

$$
\left\langle\mathrm{r}^{\mathrm{n}}\right\rangle=\mathrm{E}\left\{\mathrm{r}^{\mathrm{n}}\right\}=\exp \left(\frac{\mathrm{n}^{2} \sigma_{\tau}^{2}}{2}\right)
$$

O estimador da probabilidade acumulada empírica associada aos valores observados $\tau_{\mathrm{j}}$, ordenados de maneira crescente $\left(\tau_{1}<\cdots<\tau_{\mathrm{k}}<\cdots<\tau_{\mathrm{N}}\right)$ é dado por:

$$
\mathrm{P}_{\mathrm{k}}=\frac{\mathrm{k}}{\mathrm{N}+1}
$$

Existem outras funções probabilísticas que podem representar satisfatoriamente a distribuição dos fatores de escala, como a logística; entretanto, depois de analisar essas distribuições sob a hipótese de que o solo pode ser considerado um objeto fractal no sentido de Mandelbrot (1983), Fuentes (1992) observou que as distribuições devem proporcionar a mesma dimensão fractal do solo.

\section{Fractais auto-similares}

Os meios similares de Miller \& Miller (1956) correspondem aos fractais auto-similares. Um estimador da dimensão fractal (D) a partir da função de distribuição de probabilidades, pode ser obtida por (Fuentes, 1992):

$$
\frac{\mathrm{E}}{\mathrm{D}}=1+2 \int_{1}^{\infty} \frac{\varpi(\mathrm{r})}{\mathrm{r}^{\mathrm{E}}} \mathrm{dr}=1+2 \int_{0}^{\infty} \mathrm{e}^{-\mathrm{E} \tau} \mathrm{p}(\tau) \mathrm{d} \tau
$$

sendo $E$ a dimensão de Euclides do espaço físico $(E=3)$ e $r$ normalizado de modo que $\omega=1 / 2$ quando $\mathrm{r}=1$, isto é $\tau=\ln (\mathrm{r})=0$.

A introdução da distribuição gaussiana definida pela Eq. 12 na Eq. 16, permite calcular-se a dimensão fractal, da seguinte maneira:

$$
\frac{E}{D}=1+\exp \left(\frac{E^{2} \sigma_{\tau}^{2}}{2}\right) \operatorname{erfc}\left(\frac{E \sigma_{\tau}}{\sqrt{2}}\right)
$$

sendo erfc(x) =1- erf(x) a função erro complementar.

Pode-se demonstrar, a partir da Eq. 17, que a dimensão fractal é tal que: $\mathrm{E} / 2 \leq \mathrm{D} \leq \mathrm{E}$. O limite inferior corresponde a $\sigma_{\tau} \rightarrow 0$ e o limite superior a $\sigma_{\tau} \rightarrow \infty$.

\section{MATERIAL E MÉTODOS}

O estudo foi realizado em duas áreas experimentais, com distintas condições de clima e solo, de aproximadamente 4 ha. A primeira, localizada na Fazenda Várzea Grande, no município de São João do Cariri, $\mathrm{PB}\left(7^{\circ} 23^{\prime} \mathrm{S}\right.$ e $\left.36^{\circ} 31^{\prime} \mathrm{O}\right)$. Este município faz parte da microrregião do Cariri Oriental, mesorregião da Borborema do Estado da Paraíba. O clima é do tipo Bsh, semiárido, caracterizando-se por ser quente e seco, com chuvas de verão, alcançando-se os índices mais baixos de precipitação do estado (Brasil, 1972). O solo é classificado como Neossolo Flúvico (EMBRAPA, 1999). A segunda, localizada no Centro de Ciências Agrárias, da UFPB, no município de Areia, PB (6 ${ }^{\circ}$ 58' S, 3541' W), está inserido na microrregião do Brejo Paraibano, caracterizada por sua elevada pluviosidade (em torno de 1500 $\mathrm{mm}$ anuais) com umidade relativa do ar da ordem de $85 \%$ e temperatura média anual de $22{ }^{\circ} \mathrm{C}$, com as mínimas atingindo menos de $15{ }^{\circ} \mathrm{C}$ nos anos mais frios. O solo é classificado como Latossolo Amarelo.

A análise granulométrica do solo foi realizada em amostras coletadas na camada superficial do solo $(0-15 \mathrm{~cm})$. As frações de argila e de silte foram determinadas por sedimentação, após dispersão com hexametafosfato de sódio, utilizando-se o método da pipeta (Loveland \& Whalley, 1991). A massa específica global do solo foi determinada utilizando-se o método do anel volumétrico (Tabela 1).

Para se estimar a condutividade hidráulica à saturação do solo, realizaram-se ensaios de infiltração com infiltrômetro de anel (15 cm de diâmetro) na superfície do solo, em 80 pontos 
distribuídos regularmente em uma malha de $25 \times 25 \mathrm{~m}$. A condutividade hidráulica à saturação do solo foi estimada utilizando-se o método proposto por Haverkamp et al. (1994).

Tabela 1. Média (m), desvio padrão (DP) e coeficiente de variação $(\mathrm{CV})$ das frações granulométricas e da massa específica global $(\rho)$ da camada superficial $(0-15 \mathrm{~cm})$ dos solos de Areia e Várzea Grande

\begin{tabular}{ccccc} 
& Areia & $\begin{array}{c}\text { Silte } \\
\%\end{array}$ & Argila & $\begin{array}{c}\rho \\
\mathrm{kg} \mathrm{dm}^{-3}\end{array}$ \\
\cline { 2 - 4 } & \multicolumn{5}{c}{ Areia } \\
m & 60,81 & 14,32 & 24,86 & 1,13 \\
DP & 4,23 & 2,29 & 4,67 & 0,09 \\
CV (\%) & 6,96 & 15,99 & 18,79 & 7,96 \\
\hline \multicolumn{5}{c}{ Várzea Grande } \\
m & 65,99 & 18,34 & 15,66 \\
DP & 11,96 & 6,71 & 6,17 & 1,42 \\
CV (\%) & 18,12 & 36,59 & 39,40 & 0,14 \\
\hline
\end{tabular}

\section{RESULTADOS E DISCUSSÃO}

As determinações da condutividade hidráulica do solo em condições de saturação, em Areia, foram realizadas em 78 pontos, enquanto em Várzea Grande, em 75 pontos. A condutividade hidráulica do solo de referência em condição de saturação, foi obtida com a Eq. 7 para ambos os solos. Os valores de $\mathrm{K}_{\mathrm{s}}^{*}$ foram 98,10 e $12,65 \mathrm{~cm} \mathrm{~h}^{-1}$, respectivamente, para os solos de Areia e Várzea Grande. Os valores do fator de escala (Eq. 8) variaram de 0,23 a 2,29 e de 0,06 a 3,67, respectivamente, para os solos de Areia e Várzea Grande. Zavattaro et al. (1999) encontraram, para um solo siltoso, valores do fator de escala que variaram de 0,26 a 3,08. O desvio padrão do logaritmo dos fatores de escala, $\sigma_{\tau}$, foram $0,411 \mathrm{e}$ 0,619 , respectivamente, para os solos de Areia e Várzea Grande.

A probabilidade acumulada empírica do logaritmo dos fatores de escala ordenados de maneira crescente, Eq. 15, é comparada com a probabilidade teórica dada pela Eq. 13, para os solos de Areia e Várzea Grande (Figura 1). A boa concordância é evidenciada para ambos os solos.

Para os valores dos logaritmos dos fatores de escala, a distribuição teórica normal foi a que se aproximou mais da distribuição observada nos dois solos (Figura 1). De acordo com o teste de Kolmogorov-Smirnov, no qual se avalia o desvio absoluto máximo entre distribuições, não há diferença estatisticamente significativa, a níveis de significância de $1 \mathrm{e}$ $5 \%$, entre a distribuição de freqüências acumuladas empírica e a distribuição teórica (Normal) dos logaritmos dos fatores de escala, para os solos (Tabela 2).

Tabela 2. Teste de Kolmogorov-Smirnov dos logaritmos dos fatores para os solos de Areia e Várzea Grande

\begin{tabular}{lcccc}
\hline \multicolumn{1}{c}{$\tau=\ln (\mathrm{r})$} & $\mathrm{n}$ & $\mathrm{F}(0)-\left.\mathrm{F}(\mathrm{t})\right|_{\mathrm{i}}<\mathrm{P}$ & $\mathrm{P}_{\mathrm{KS}}(0,05)^{*}$ & $\mathrm{P}_{\mathrm{KS}}(0,01)^{*}$ \\
\hline Areia & 78 & 0,083 & 0,154 & 0,184 \\
Várzea Grande & 75 & 0,119 & 0,157 & 0,188 \\
\hline
\end{tabular}

* Probabilidade acumulada teórica a 95 e $99 \%$ de intervalo de confiança
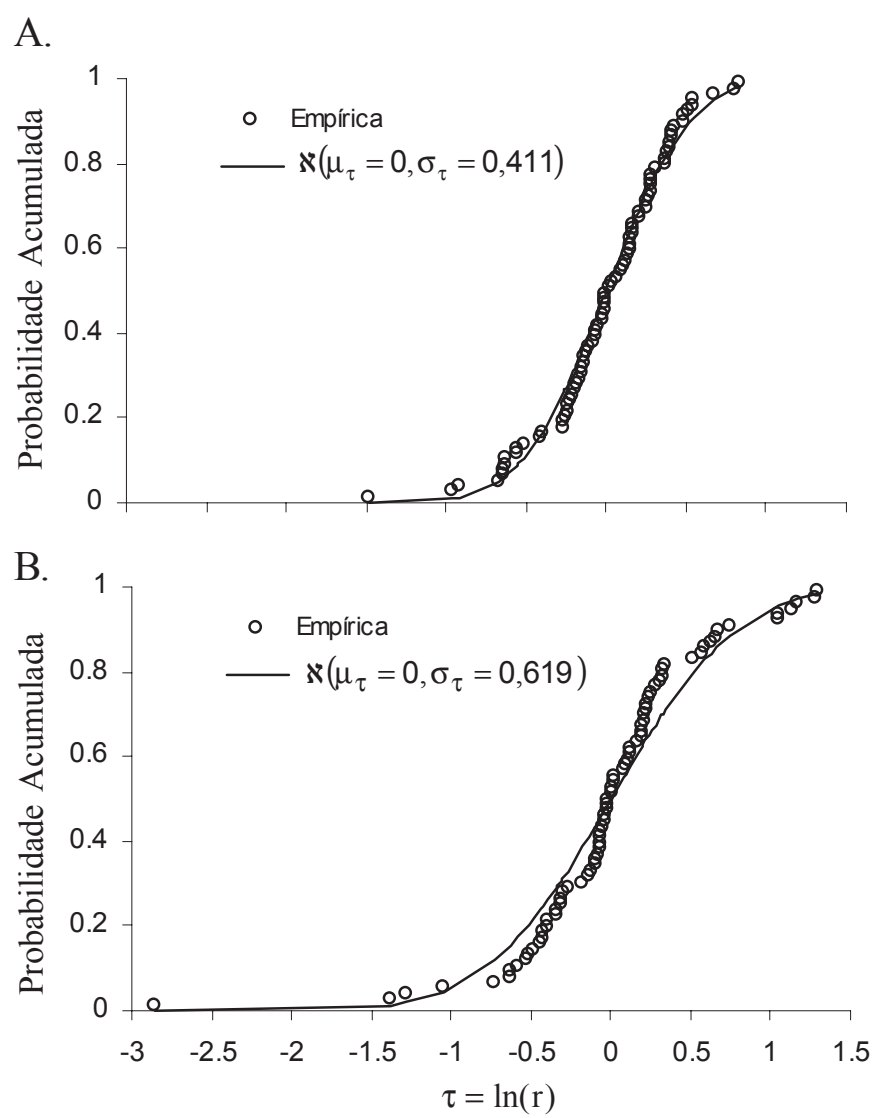

Figura 1. Probabilidade empírica e teórica (Normal) acumulada dos fatores de escala, $\tau=\ln (\mathrm{r}):$ A) Latossolo Amarelo e B) Neossolo Flúvico

Vários estudos (Kosugi \& Hopmans, 1998; Zavattaro et al., 1999; Comegna et al., 2000) têm mostrado que a distribuição de freqüência do fator de escala segue uma distribuição log-normal. A estimativa da média, da variância e do coeficiente de variação do fator de escala (distribuição log-normal) dos solos de Areia e Várzea Grande, é apresentada na Tabela 3.

Tabela 3. Média (m), Variância (V) e coeficiente de variação (CV) do fator de escala (distribuição log-normal) dos solos de Areia e Várzea Grande

\begin{tabular}{ccc}
\hline & Areia & Várzea Grande \\
\hline $\mathrm{m}$ & 1,09 & 1,21 \\
$\mathrm{~V}$ & 0,22 & 0,69 \\
$\mathrm{CV}(\%)$ & 43 & 68 \\
\hline
\end{tabular}

A dimensão fractal, D, foi obtida por intermédio da Eq. 17 $(E=3)$ e seus valores foram de 2,047 e 2,214, respectivamente, para os solos de Areia e Várzea Grande. A dimensão fractal do solo de Várzea Grande foi maior que a do solo de Areia visto que, texturalmente, o de Várzea Grande apresenta maior variabilidade que o solo de Areia, apresentando coeficientes de variação da ordem de duas vezes maior para as frações areia, silte e argila, que o de Areia (Tabela 1). Para o solo de Areia, a maioria dos pontos amostrados, $83,95 \%$, classifica-se como franco argilo arenoso, enquanto para o solo de Várzea Grande a maioria dos pontos amostrados está distribuída em três classes texturais, $52,5 \%$ franco arenoso, $23,75 \%$ franco 
argilo arenoso e 17,75\% areia franca, indicando diferentes graus de fragmentação. A medida em que a dimensão fractal se aproxima da dimensão euclidiana $(\mathrm{E}=3)$ existe uma faixa mais ampla de escalas, na qual o solo apresenta diferentes graus de fragmentação (Rieu \& Sposito, 1991).

\section{CONCLUSÕES}

1. Para ambos os solos, o logaritmo dos fatores de escala que intervém nos fractais auto-similares segue uma distribuição de Gauss ou distribuição normal.

2. A função de distribuição probabilística permitiu estimarse a dimensão fractal do meio poroso que representa os solos estudados.

3. Os solos têm dimensão fractal de 2,047 e 2,214, respectivamente, para o Latossolo Amarelo (Areia) e o Neossolo Flúvico (Várzea Grande).

\section{LITERATURA CITADA}

Brasil. Ministério da Agricultura. Equipe de pedologia e fertilidade do Solo. Divisão de Agrologia - SUDENE. Levantamento exploratório - Reconhecimento de solos do Estado da Paraíba. Rio de Janeiro. 1972. 670p. Boletim Técnico, 15

Braud, I.; Antonino, A.C.D.; Vauclin, M. A stochastic approach to studying the influence of the spatial variability of soil hydraulic properties on surface fluxes, temperature and humidity. Journal of Hydrology, Amsterdam, v. 165, p. $283-$ 310, 1995.

Comegna, V.; Damiani, P.; Sommella, A. Scaling the saturated hydraulic conductivity of a vertic ustorthens soil under conventional and minimum tillage. Soil \& Tillage Research, Madison, v.54, p.1-9, 2000.

EMBRAPA - Empresa Brasileira de Pesquisa Agropecuária. Centro Nacional de Pesquisa de Solos. Sistema brasileiro de classificação de solos. Brasília: EMBRAPA - Serviço de Produção da Informação; Rio de Janeiro, EMBRAPA solos, 1999. 412p.

Fuentes, C. Approche fractale des transferts hydriques dans les sols non-saturés. Grenoble: Universidade Joseph Fourier, 1992. 267p. Tese Doutorado
Haverkamp, R.; Ross, P.J.; Smettem, K.R.J.; Parlange, J.Y. Three dimensional analysis of infiltration from the disc infiltrometer. 2. Physically based infiltration equation. Water Resources Research, Washington, v.30, p.2931-2935, 1994.

Hopmans J. W. A comparison of various methods to scale soil hydraulic properties. Journal of Hydrology, Amsterdam, v. 93, p. 241-256, 1987.

Kosugi, K.; Hopmans, J.W. Scaling water retention curves for soils with lognormal pore-size distribution. Soil Science Society America Journal, Madison, v.62, p.1496-1505, 1998.

Loveland, P.J.; Whalley, R.W. Particle size analysis. In : Smith K.A; Mullins C.E., (ed). Soil analysis - Physical methods. New York : Marcel Dekker, Inc, 1991. p.271-328.

Mandelbrot, B.B. The fractal geometry of nature. San Francisco: Freeman, 1983, 468 p.

Miller, E.E.; Miller, R.D. Physical theory for capillary flow phenomena. Journal of Applied Physics, Boston, v.27, p.324332,1956

Namuche, R.; Fuentes, C.; Rendón, L.; Mercado, R. Análisis probabilístico del espaciamiento entre drenes subterráneos parcelarios. In: Memórias do Congresso Latinoamericano de Hidráulica, 17, Guayaquil, Equador, v.5, 1996. p.413-426.

Nielsen, D.R.; Biggar, J.W.; Erh, K.T. Spatial variability of field measured soil-water properties. Hilgardia, Oakland, California, v.42, p.215-259, 1973.

Rieu, M.; Sposito, Y.G. Fractal fragmentation, soil porosity and soil water properties: I. Theory. Soil Science Society America Journal, Madison, v.55, p.1231-1238, 1991.

Vauclin, M. Méthodes d'étude de la variabilité spatiale des propriétés d'un sol. In: Variabilité spatiale des processus de transfert dans les sols. Colloque S.H.F.-I.N.R.A., Avignon, França, v.15, 1982. p.9-13.

Warrick, A.W.; Amoozegard-Fard, A. Infiltration and drainage calibration using a similar media concept. Water Resources Research, Washington, v. 13, p.355-362, 1979.

Zavattaro, L.; Jarvis, N.; Persson, L. Use of similar media scaling to characterize spatial dependence of near-saturated hydraulic conductivity. Soil Science Society America Journal, Madison, v.63, p.486-492, 1999. 